\title{
Social Machines
}

\section{Claude Draude ${ }^{1}$ Christian Gruhl' ${ }^{1}$ Gerrit Hornung ${ }^{1}$ Jonathan Kropf ${ }^{1}$ Jörn Lamla ${ }^{1}$ Jan Marco Leimeister ${ }^{1}$. Bernhard Sick ${ }^{1} \cdot$ Gerd Stumme ${ }^{1}$}

Angenommen: 4. Oktober 2021 / Online publiziert: 11. November 2021

(c) Der/die Autor(en) 2021

\section{Zusammenfassung}

Social Machines sind ein Paradigma für die Gestaltung soziotechnischer Systeme, die unter Verwendung von Web- und Plattformlösungen das Potenzial digitaler Technologien mit der Eigenlogik sozialer Interaktion, Organisation und Strukturbildung auf neue Weise zusammenführen. Im Folgenden diskutieren wir das Paradigma der Social Machine aus den Perspektiven der Informatik, der Wirtschaftsinformatik, der Soziologie und des Rechts, um Orientierungspunkte für seine Gestaltung zu identifizieren. Der Begriff ist in der Literatur jedoch bisher nicht abschließend definiert sondern nur durch Beispiele illustriert.

In diesem Artikel stellen wir zunächst die folgende Definition zur Diskussion: Social Machines sind soziotechnische Systeme, in denen die Prozesse sozialer Interaktion hybrid zwischen menschlichen und maschinellen Akteuren ablaufen und teilweise algorithmisiert sind. Im Anschluss beleuchten wir drei aktuelle, sich gegenseitig bedingende Entwicklungen von Social Machines: die immer stärkere Verschmelzung von Sozialiät und Maschine, die Vermessung von Nutzeraktivitäten als Grundstoff gesellschaftlichen Zusammenhalts und die zunehmende Algorithmisierung gesellschaftlicher Prozesse. Abschließend diskutieren wir, dass eine teilhabeorientierte, demokratischen Werten folgende Gestaltung von Social Machines die Perspektiven der Nutzungsakzeptanz, der gesellschaftlichen Akzeptabilität und der nachhaltigen Wirtschaftlichkeit adressieren und umsetzen muss.

Unter Social Machines werden heute soziotechnische Systeme verstanden, die unter Verwendung von Web- und Plattformlösungen das Potenzial digitaler Technologien mit der Eigenlogik sozialer Interaktion, Organisation und Strukturbildung auf neue Weise zusammenführen. Entsprechende Experimente reichen von der globalen Volksenzyklopädie Wikipedia über neue Tools der politischen Willensbildung wie Liquid Democracy bis zu Crowdfunding-Plattformen. Es handelt sich um ein Paradigma gegenwärtiger soziotechnischer Gestaltung, das sich in einer Reihe von IT-Projekten niederschlägt. Geprägt wurde der Begriff von Tim BernersLee, dem Erfinder des World Wide Web: „Real life is and must be full of all kinds of social constraint - the very processes from which ,society ' arises. Computers can help if we use them to create abstract social machines on the Web: processes in which the people do the creative work and the

Gerd Stumme

stumme@cs.uni-kassel.de

1 Wissenschaftliches Zentrum für Informationstechnik-Gestaltung (ITeG), Universität Kassel, Kassel, Deutschland machine does the administration" [2, S. 172]. Allerdings zeigt die Praxis, dass sich Kreativität und Administration nicht so einfach auf Mensch und Maschine aufteilen lassen. Auch in aktuellen Projekten (etwa [14] und SOCIAM ${ }^{1}$ [36]) kommt das Paradigmatische dieser Form digitaler Gesellschaftsgestaltung nicht klar zum Ausdruck; die Autoren verwenden stattdessen eine (nichtabschließende) Liste von Beispielen zur Begriffsabgrenzung.

Im Folgenden diskutieren wir das Paradigma der Social Machine aus den Perspektiven der Informatik, der Wirtschaftsinformatik, der Soziologie und des Rechts, um Orientierungspunkte für seine Gestaltung zu identifizieren. Hierzu stellen wir die folgende Definition zur Diskussion:

Social Machines sind soziotechnische Systeme, in denen die Prozesse sozialer Interaktion hybrid zwischen menschlichen und maschinellen Akteuren ablaufen und teilweise algorithmisiert sind.

Hierbei verstehen wir unter einem soziotechnischen System ein komplexes Gefüge, welches Menschen, Hard- und Software, organisationale und soziale Prozesse für gegebe-

\footnotetext{
${ }^{1}$ https://www.sociam.org/.
} 
ne Aufgaben oder Ziele miteinander interagieren lässt [22]. Prozesse sozialer Interaktion sind sich dynamisch ändernde Abfolgen sozialer Aktionen zwischen Individuen und/oder Gruppen. Unter ihrer Hybridität schließlich verstehen wir, dass an diesen Prozessen inhärent sowohl maschinelle als auch menschliche Akteure wesentlich beteiligt sind.

Die jüngeren Entwicklungen von Social Machines weisen insbesondere in drei Richtungen: (1) Durch das Mobilwerden der Webtechnologie, aber auch durch lernende Bots und Fortschritte im Bereich der künstlichen Intelligenz verschmelzen Sozialität und Maschine zunehmend [10]. Die menschlichen und nichtmenschlichen Komponenten der Social Machine sind folglich immer schwerer voneinander $\mathrm{zu}$ unterscheiden und zu trennen, was als paradigmatischer Trend zur fortschreitenden Hybridisierung der Social Machine bezeichnet werden kann. (2) Eine starke Ausweitung erfährt die Algorithmisierung gesellschaftlicher Prozesse und letztlich der Sozialität selbst [35], indem bspw. Entscheidungen und Empfehlungen in immer mehr Lebensbereichen (vom Onlineshopping bis zum autonomen Fahren) an Algorithmen delegiert oder durch diese unterstützt werden, die ihrerseits über Rückkopplungsschleifen mit dem sozialen Leben verbunden sind. Das wirft die Frage auf, ob das Paradigma der Social Machine zukünftig bloß begrenzte soziale Bereiche algorithmisch unterstützt oder das gesellschaftliche Leben im Ganzen algorithmisiert [1] und damit überformt. (3) Schließlich wird die Social Machine der Zukunft noch stärker durch die Vermessung von Nutzeraktivitäten getrieben sein. Daten werden damit mehr und mehr zum Grundstoff des sozialen Zusammenhalts [19, 29] und ersetzen andere Quellen gesellschaftlicher Bindung und Organisation (z. B. Predictive Policing im Unterschied zur klassischen Strafverfolgung).

\section{(1) Die Hybridisierung der Social Machine}

Hybride Intelligenz ist die Fähigkeit, komplexe Ziele durch die Kombination menschlicher und maschineller Intelligenz zu erreichen, mit besseren Ergebnissen als sie separat möglich gewesen wären, und unter kontinuierlicher Verbesserung durch gegenseitiges Voneinanderlernen [7]. Im Fokus stehen hierbei dynamische Umgebungen und Probleme, deren Lösung domänenspezifisches Wissen bedürfen und die keine spezifische Grundwahrheit (Ground Truth) besitzen. So kann einerseits das System den Menschen trainieren (AI in the Loop) und andererseits der Mensch das System trainieren (Human in the Loop). Es können Probleme gelöst werden, deren Lösungen bisher typischerweise dem menschlichen Intellekt vorbehalten waren und beispielsweise strategische oder politische Entscheidungen betreffen [7, 39].
Zur Realisierung von hybrider Intelligenz müssen zukünftige soziotechnische Systeme wie Social Machines so gestaltet werden, dass ihre technischen Systeme über 3 Haupteigenschaften verfügen, die sich zusammengefasst als kollaborativ-interaktiv lernend (engl. Collaborative Interactive Learning, CIL) bezeichnen lassen [3, 37]: CIL-Systeme müssen über ihren gesamten Lebenszyklus lernen. Das bedeutet, dass sie ihre eigene Wissensbasis in einer selbstorganisierten Weise sowie ihren Lernprozess kontinuierlich verbessern. Darüber hinaus müssen sie andere Entitäten (Mensch/Maschine) in ihren Lern-/Arbeitsprozess einbeziehen und mit dieser heterogenen, zeitveränderlichen Menge an Entitäten kollaborieren, um ihre und gemeinsame Ziele zu erreichen.

Wissenschaftliche Analysen, normative Bewertungen und rechtliche Regulierung müssen der Hybridität der Social Machine Rechnung tragen. Hier wird bisher zum Teil der zweite Schritt vor dem ersten gemacht und über Herausforderungen KI-basierter Entscheidungsfindungen diskutiert, in denen die menschlichen Beiträge völlig in den Hintergrund treten. Stattdessen werden beispielsweise Regulierer und Rechtspraxis auf absehbare Zeit gerade die Probleme lösen müssen, die sich aus dem hybriden Charakter, also aus der Interaktion und Vermischung menschlicher und maschineller Intelligenz ergeben.

Die Neuverteilung der Arbeitsteilung von menschlicher Intelligenz und maschinellen Verfahren im Zuge der Social Machine stellt einen Perspektivwechsel dar, der die Mensch-Computer-Interaktion (MCI) vor Herausforderungen stellt. So fordert das Verständnis einer hybriden Intelligenz als soziotechnisches Ensemble die MCI heraus, die Trennung zwischen Mensch und Maschine grundlegend zu überdenken und neue Konzepte zu entwickeln. Für verteilte, hybride intelligente Systeme werden Frameworks entwickelt, die menschliche ebenso wie nichtmenschliche Akteure und erweiterte Kontexte in den Blick nehmen - hierzu werden Ansätze aus den Science \& Technology Studies und dem Posthumanismus integriert [5, 8]. Auch Agency (Handlungsfähigkeit) muss in Vernetzung und hybride gedacht werden [21]. Für die Nutzenden von Social Machines wirft die Hybridisierung Fragen der Nachvollziehbarkeit vermischter Informations- und Wissensaustauschprozesse sowie der aktiven Teilhabe Nutzender (oder auch Betroffener) an der Gestaltung der Social Machines auf. Unter Explainable Artifical Intelligence (xAI) werden Forschungsansätze gefasst, die auf Nachvollziehbarkeit maschineller Entscheidungsprozesse setzen $[6,33]$ und Transparenz, Vertrauen, Fairness oder Accountability von intelligenten Systemen gewährleisten wollen [30]. Angelehnt an die UserExperience-Forschung (UX) stellt die Algorithmic-Experience-Forschung (AX) das Nutzererlebnis in realweltlichen Kontexten in den Mittelpunkt. Studien zeigen, dass einem Großteil der Endanwender die Verwendung von KI entwe- 
der überhaupt nicht bewusst ist, oder dass ein Verständnis von Funktionsweisen und Technikfolgen nicht oder nur unzureichend vorliegt [11, 16, 28].

Eine weitere Perspektive auf ,soziale Maschine(n)“ bietet die Soziologie. Hiernach gilt es, in einem offenen Suchprozess zunächst die Ausprägungen und Transformationen medial-kommunikativer Infrastrukturen zu untersuchen sowie ihre gesellschaftlichen Bedingungen und Folgen zu reflektieren. Unter dem Begriff der sozialen Maschine im Singular werden die paradigmatischen Verschiebungen in den Blick genommen die sich mit der Ausbreitung des ganzen Spektrums hybrider, Menschen und Maschinen in digitalen Infrastrukturen in Kooperation bringender Anwendungen für das gesellschaftliche Handeln und Zusammenleben ergeben.

\section{(2) Algorithmisierung sozialer Prozesse}

Menschliche Entscheidungen und Handlungen werden zunehmend algorithmisch unterstützt oder von Algorithmen ganz übernommen. In einer gesellschaftstheoretischen Perspektive lässt sich dieser Trend auf die langfristigen Wandlungen beziehen, die das Maschinelle als infrastrukturelles Moment in der Gesellschaft erfährt, etwa auf Bedeutungsschübe sozialphysikalischen Denkens und Gestaltens, die mit der Industrialisierung ansetzen [18, 27, 32] und durch den Möglichkeitsraum digitaler Technik heute neu belebt werden [26], aber auch kritisch zu untersuchen sind [41]. In diesem Zusammenhang stellt sich die Frage, inwiefern Social Machines das Paradigma kybernetischer Feedbackschleifen verallgemeinern und vertiefen [24] oder aber eine Dynamik der rekursiven Anpassung, Angleichung und Abschließung von sozialen Prozessmustern zu vermeiden helfen.

Wenn in immer mehr sozialen Bereichen gesellschaftliche Prozesse durch algorithmisierte Rückkopplungen geprägt sind, so muss diese Verschleifung bei der Analyse hybrider Lernprozesse, der Entwicklung angemessener rechtlicher Regulierungsvorschläge und der Achtsamkeit für neue gesellschaftliche Ungleichheiten Berücksichtigung finden.

Für die Algorithmisierung sozialer Prozesse gemäß des CIL-Ansatzes werden aus Sicht der Informatik geeignete Mechanismen der KI, insbesondere des Maschinellen Lernens (ML) benötigt. Insbesondere haben dabei die Gebiete Human-in-the-Loop ML [23], Human-Centered AI und Interactive ML [15, 38] eine hohe Bedeutung. Während in anderen Bereichen (z.B. Autonomous Learning) untersucht wird, wie technische Systeme hochgradig automatisiert oder völlig autonom lernen, geht es hier darum, Menschen effizient und effektiv (aus Sicht der KI) in den maschinellen Lernprozess einzubeziehen. Eine Schlüsselrolle kommt dabei dem Aktiven Lernen (AL) [34] zu, das beispielsweise versucht, ausgehend von einer Menge nichtannotierter Daten die für ein überwachtes Lernen erforderlichen Annotationen von menschlichen Experten so zu erhalten, dass mit möglichst wenig Fragen (und damit Annotationen) die Performanz eines ML-Modells maximiert wird. Dieses konventionelle aktive Lernen trifft jedoch häufig Annahmen (Annotatoren sind immer verfügbar, liefern immer eine korrekte Antwort, beantworten immer denselben Typ von Fragen usw.), die für Anwendungen in Social Machines auf der Basis von CIL nicht realistisch sind. Somit stellen sich im Bereich des Aktiven Lernens mit unsicheren (möglicherweise fehlerhaften) Annotatoren, unterschiedlichen Fragetypen und mehr oder wenigen komplexen Kostenschemen für die Auswahl von zu bewertenden Daten, Fragen und Annotatoren viele algorithmische Herausforderungen. Diese zu lösen, ist umso schwieriger, wenn die MLModelle aus dem Bereich des Deep Learnings kommen, das typischerweise auf großen Datenmengen basiert (z. B. im Bereich der Objekterkennung in Bildern). Außerdem beschäftigt sich Aktives Lernen bisher nicht mit dem Aspekt einer Kooperation oder gar Kollaboration von Menschen in diesem Lernprozess. Collaborative Interactive Learning wird Aktives Lernen aber auch mit weiteren Lernparadigmen kombinieren müssen, beispielsweise Transferlernen, um auf früheres Wissen zurückzugreifen, Kollaboratives Lernen, um in einem verteilten System das Wissen anderer zu nutzen oder Verstärkendes Lernen, um neues Wissen explorativ zu generieren und Lernmechanismen selbst zu wählen und geeignet anzustoßen. Daher müssen lernende Systeme ihren eigenen Zustand (unter Berücksichtigung von Unsicherheiten) geeignet bewerten können, eine Art technische „Self-Awareness“.

Zur vorausschauenden Adressierung rechtlicher Konflikte bei der Übertragung sozialer Prozesse an Algorithmen wird man um Vorgaben für die Protokollierung menschlicher und maschineller Verantwortungsbeiträge nicht herumkommen. Aber die klare Trennung dieser Sphären, wie sie z. B. der Regelung in $\S 63$ a StVG für hoch- oder vollautomatisierte Fahrfunktionen zugrunde liegt, wird sich perspektivisch nicht durchhalten lassen. Ähnliche Herausforderungen stellen sich für rechtliche Transparenzregeln. Diese können Transparenz in beide Richtungen verlangen: Hinsichtlich des Verantwortungsbeitrags automatisierter Systeme, aber auch desjenigen menschlicher Akteure, weil es je nach Konstellation ein legitimes Interesse geben kann, auch letzteres zu erfahren. Allerdings wird auch hier Transparenz mutmaßlich an umso stärkere Grenzen stoßen, je größer der Grad der Hybridität ausfällt. Ob und - wenn ja - welche Grenzen das Recht für (ggf. dem Grad nach intransparente) rechtserhebliche Entscheidungen einer hybriden Social Machine stellen sollte, ist bislang kaum diskutiert, weil sich die rechtliche Regelung ,,roter Linien“ wie in Art. 22 DSGVO 
oder $§ 35 \mathrm{a}$ VwVfG auf vollständig automatisierte Entscheidungen beschränkt.

Soziale Ungleichheitsforschung macht sichtbar, dass verschiedene Menschen und Personengruppen unterschiedlich durch algorithmische Automationsprozesse betroffen sind $[13,25]$. Avancierte Algorithmen, die auf einer Einbindung von Menschen und Echtzeitfeedbacks beruhen, bergen zudem die Gefahr, subtile Formen der Beeinflussung oder des „Hypernudgings“ [40] zu befördern, die die autonome Bewertungs- und Entscheidungskompetenz alleine schon durch die Verringerung produktiver Diskrepanzerfahrungen aushöhlen [17]. Wenn wir von einer Algorithmisierung gesellschaftlicher Prozesse sprechen, muss berücksichtigt werden, wie zukünftige Social Machines soziale und ökonomische Spaltung und gesellschaftliche Ungleichgewichte nicht verstärken, sondern integrierend wirken.

\section{(3) Daten als Grundstoff sozialen Zusammenhalts}

Der spezifische, hybride Charakter von Social Machines führt dazu, dass schon bei ihrer Erforschung und Implementierung, noch mehr aber im späteren Einsatz umfassende Daten über Eigenschaften und Verhalten anfallen. Weder menschliches noch Maschinelles Lernen ist ohne Daten denkbar, und die auf Basis des Erlernten gefällten Entscheidungen und sozialen Handlungen produzieren unweigerlich neue Daten. Social Machines stehen damit inmitten aktueller Diskussionen um den Schutz personenbezogener Daten, Datensouveränität und Data Governance, in denen Persönlichkeitsrechte, wirtschaftliche Verwertungsinteressen und staatliche Gestaltungsansprüche immer neu aufeinandertreffen.

Die Herausforderung liegt in der Entwicklung angemessener Regulierungsstrategien, die auf Hybridisierung und Algorithmisierung reagieren und die mit ihnen verbundenen sozialen Konflikte adressieren. Dabei könnte sich der bisherige datenschutzrechtliche Rahmen für den Umgang mit Trainingsdaten als zu enges Korsett erweisen, wenn der system- oder sogar betreiberübergreifende Austausch zum Lernen seltener, schadensträchtiger Ereignisse erforderlich ist. Hier werden sich übergeordnete, gesellschaftliche Interessen ergeben, die für eine Datennutzung sprechen - in aller Regel allerdings nicht in personenbezogener Form. Außerdem bieten die datenschutzrechtlichen Betroffenenrechte, die Möglichkeiten kollektiven Rechtsschutzes und die Instrumente zur Risikoadressierung (Datenschutzfolgenabschätzung, Datenschutzbeauftragte, technische und organisatorische Maßnahmen der Risikominimierung) wichtige Partizipations- und Gestaltungsansätze.

Verfahren Maschinellen Lernens, die (notwendigerweise) Datensammlungen der Vergangenheit nutzen, überneh- men auch deren Wertsetzungen und Einordnungen. Werden hieraus Vorhersagen für die Zukunft abgeleitet kann das problematisch sein. Es scheint, dass algorithmische Vorhersagen inhärent konservativ/konservierend wirken und nicht geeignet sind, um sozialen Wandel zu modellieren oder Innovationen anzustoßen. Bias bzw. die Verfestigung von Ungleichheiten kann z. B. daher stammen, dass diskriminierende oder rassistische Klassifizierungen (unreflektiert oder unwissentlich) übernommen werden oder auch, dass bestimmte Menschen (oder auch Wissensobjekte, Kategorisierungen, Merkmale) im Datensatz nicht vorkommen [20]. In der Nutzung von Social Machines treten solche Setzungen dann zumeist als technologische Eigengesetzlichkeiten auf (Code is Law) bzw. sind für die Nutzenden nicht verständlich oder sichtbar [9]. Diesem muss durch entsprechende Gestaltungsverfahren entgegengewirkt werden.

Verfahren Maschinellen Lernens können jedoch auch dazu dienen, gesellschaftliche Ungleichheit und ungleiche Teilhabe überhaupt erst sichtbar zu machen [4]. Die datenförmige Erfassung des gesamten Lebensalltags wird zudem unter dem Stichwort der „Big Data“ häufig mit dem Versprechen verbunden, „Muster“ im sozialen Zusammenleben zu erkennen [24] und darüber Stellschrauben zur effektiven Gesellschaftssteuerung zu identifizieren [26]. Aus soziologischer Sicht gilt es zu untersuchen, welche gesellschaftlichen Effekte sich aus dieser zunehmenden Verdatung des Lebens ergeben [12, 31].

\section{Gestaltung von Social Machines als interdisziplinäre Herausforderung}

Für eine teilhabeorientierte, demokratischen Werten folgende Gestaltung von Social Machines müssen Perspektiven der Nutzungsakzeptanz, der gesellschaftlichen Akzeptabilität und der nachhaltigen Wirtschaftlichkeit adressiert und umgesetzt werden. Um Gestaltungskriterien zu entwickeln, die gesellschaftlich wünschenswert, technisch umsetzbar und wirtschaftlich sinnvoll sind, müssen die skizzierten Herausforderungen der Hybridisierung, der Algorithmisierung von Gesellschaft und der Daten in ihrer Verschränkung interdisziplinär angegangen werden. Vor diesem Hintergrund stellt der Begriff der Social Machine einen grundlagen- und gesellschaftstheoretischen sowie wirtschaftlichen Reflexionshorizont für die zukünftige Konfiguration von Sozialität und Digitalität bereit, an den gestaltungsorientierte Forschungsansätze anschließen können.

Funding Open Access funding enabled and organized by Projekt DEAL.

Open Access Dieser Artikel wird unter der Creative Commons Namensnennung 4.0 International Lizenz veröffentlicht, welche die Nut- 
zung, Vervielfältigung, Bearbeitung, Verbreitung und Wiedergabe in jeglichem Medium und Format erlaubt, sofern Sie den/die ursprünglichen Autor(en) und die Quelle ordnungsgemäß nennen, einen Link zur Creative Commons Lizenz beifügen und angeben, ob Änderungen vorgenommen wurden.

Die in diesem Artikel enthaltenen Bilder und sonstiges Drittmaterial unterliegen ebenfalls der genannten Creative Commons Lizenz, sofern sich aus der Abbildungslegende nichts anderes ergibt. Sofern das betreffende Material nicht unter der genannten Creative Commons Lizenz steht und die betreffende Handlung nicht nach gesetzlichen Vorschriften erlaubt ist, ist für die oben aufgeführten Weiterverwendungen des Materials die Einwilligung des jeweiligen Rechteinhabers einzuholen.

Weitere Details zur Lizenz entnehmen Sie bitte der Lizenzinformation auf http://creativecommons.org/licenses/by/4.0/deed.de.

\section{Literatur}

1. Bächle TC (2015) Mythos Algorithmus. Die Fabrikation des computerisierbaren Menschen. Springer, Wiesbaden

2. Berners-Lee T (1999) Weaving the web: the original design and ultimate destiny of the world wide web by its inventor. Harper, New York

3. Calma A, Leimeister JM, Lukowicz P, Oeste-Reiß S, Reitmaier T, Schmidt A, Sick B, Stumme G, Zweig KA (2016) From active learning to dedicated collaborative interactive learning. Proc. Intl. Conf. on Architecture of Computing Systems (ARCS 2016), Nuremberg, Germany, S 1-8

4. Chun W (2021) Discriminating data. How Big Data and machine learning encode discrimination and create agitated clusters of comforting rage. MIT Press, Cambridge

5. Coulton P, Lindley JG (2019) More-than human centred design: considering other things. Des J 22:463-481

6. Danding W, Qian Y, Ashraf A, Brian YL (2019) Designing theorydriven user-centric explainable. AI. Proc. 2019 CHI Conf. on Human Factors in Computing Systems. Paper, Bd. 601. Association for Computing Machinery, New York, S 1-15

7. Dellermann D, Ebel P, Söllner M, Leimeister JM (2019) Hybrid intelligence. Bus Inf Syst Eng 61(5):637-643

8. Draude C (2020) "Boundaries do not sit still". From interaction to agential intra-action in HCI. In: Kurosu M (Hrsg) Human-computer interaction. Design and user experience 22nd Intl. Conf., HCII 2020. Thematic Area, HCI 2020. Springer, Cham, S 20-32

9. Draude C, Klumbyte G, Lücking P, Treusch P (2020) Situated algorithms: a sociotechnical systemic approach to bias. Online Inf Rev 44(2):325-342. https://doi.org/10.1108/OIR-10-2018-0332

10. Engemann C, Sudmann A (Hrsg) (2017) Machine Learning - Medien, Infrastrukturen und Technologien der Künstlichen Intelligenz. transcript, Bielefeld

11. Eslami M, Rickmann A, Vaccaro K, Aleyasen A, Vuong A, Karahalios K, Hamilton K, Sandvig C (2015) I always assumed that I wasn't really that close to (her): reasoning about invisible algorithms in news feeds. Proc. 33rd Annual ACM Conf. on Human Factors in Computing Systems. ACM, New York, S 153-162

12. Espeland W, Stevens M (2008) A sociology of quantification. Eur J Sociol 49(3):401-436

13. Eubanks V (2018) Automating inequality: how high-tech tools profile, police, and punish the poor. St. Martin's Press, New York

14. Hendler J, Mulvehill AM (2016) Social machines-the coming collision of artificial intelligence, social networking, and humanity. Apress, New York

15. Holzinger A (2016) Interactive machine learning (iML). Informatik Spektrum 39(1):64-68
16. Klumbyte G, Lücking P, Draude C (2020) Reframing AX with critical design. The potentials and limits of algorithmic experience as a critical design. Proc. 11th Nordic Conf. on Human-Computer Interaction. ACM, New York

17. Lamla J (2019) Selbstbestimmung und Verbraucherschutz in der Datenökonomie. Aus Polit Zeitgesch 69(24-26):49-54

18. Marx K (1962) Das Kapital. Kritik der politischen Ökonomie. Erster Band: Der Produktionsprozess des Kapitals. Dietz, Berlin ([1867])

19. Mau S (2017) Das metrische Wir. Über die Quantifizierung des Sozialen. Suhrkamp, Berlin

20. Mehrabi N, Morstatter F, Saxena N, Lerman K, Galstyan A (2019) A survey on bias and fairness in machine learning. arXiv: 1908.09635

21. Moradi M, Moradi M, Bayat F, Toosi AN (2019) Collective hybrid intelligence: towards a conceptual framework. Int J Crowd Sci 3(2): 198-220

22. Mumford E (1987) Sociotechnical systems design-evolving theory and practice. In: Bjerknes G, Ehn P, Kyng S (Hrsg) Computers and democracy: a Scandinavian challenge. Avebury, , S 59-77

23. Munro R (2019) Human-in-the-loop machine learning: active learning, annotation, and human-computer interaction. Manning, Shelter Island

24. Nassehi A (2019) Muster. Theorie der digitalen Gesellschaft. C.H. Beck, München

25. O'Neil C (2016) Weapons of math destruction: how big data increases inequality and threatens democracy. Broadway Books, New York

26. Pentland A (2014) Social physics. How good ideas spread-the lessons from a new science. Scribe, Brunswick, London

27. Quételet A (1914) Soziale Physik oder Abhandlung über die Entwicklung der Fähigkeiten des Menschen. 2 Bd. Fischer, Jena

28. Rader E, Cotter K, Cho J (2018) Explanations as mechanisms for supporting algorithmic transparency. Proc. $2018 \mathrm{CHI}$ Conf. on $\mathrm{Hu}-$ man Factors in Computing Systems. ACM, New York, S 1-13

29. Reichert R (Hrsg) (2014) Big Data. Analysen zum digitalen Wandel von Wissen, Macht und Ökonomie. transcript, Bielefeld

30. Ribera M, Lapedriza A (2019) Can we do better explanations? A proposal of user-centered explainable AI. Joint Proc. of the ACM IUI 2019 Workshops, Los Angeles. ACM, New York

31. Ringel L, Espeland W, Sauder M, Werron T (Hrsg) (2021) World of rankings. Emerald, Bingley

32. de Saint-Simon C (1977) Ausgewählte Schriften. Akademie-Verlag, Berlin ([ca. 1817])

33. Samek W, Wiegand T, Müller K-R (2017) Explainable artificial intelligence: understanding, visualizing and interpreting deep learning models. ITU Journal: ICT Discoveries Special Issue, Bd. 1

34. Settles B (2009) Active learning literature survey. Technical report. https://minds.wisconsin.edu/handle/1793/60660 (College of Letters and Science, University of Wisconsin-Madison). Zugegriffen: 9.11.2021

35. Seyfert R, Roberge J (Hrsg) (2016) Algorithmuskulturen. Über die rechnerische Konstruktion der Wirklichkeit. transcript, Bielefeld

36. Shadbolt N, De Roure D, Hall W, O'Hara K (2019) The theory and practice of social machines. Springer, Cham

37. Sick B, Oeste-Reiß S, Schmidt A, Tomforde S, Zweig AK (2018) Collaborative interactive learning. Informatik Spektrum 41(1):52-55

38. Teso S, Hinz O (2020) Challenges in interactive machine learning. Künstl Intell 34(2):127-130

39. Traumer F, Oeste-Reiß S, Leimeister JM (2017) Towards a future reallocation of work between humans and machines - taxonomy of tasks and interaction types in the context of machine learning

40. Yeung K (2017) 'Hypernudge': Big Data as a mode of regulation by design. Inf Commun Soc 20(1):118-136

41. Zuboff S (2018) Das Zeitalter des Überwachungskapitalismus. Campus, Frankfurt/M., New York 